\title{
Selbstverpflichtung der DGN zu Transparenz, Ethik und Vermeidung von - Interessenkonflikten
}

Die Deutsche Gesellschaft für Nuklearmedizin (DGN) ist die einzige wissenschaftlich medizinische Fachgesellschaft, die das Fachgebiet der Nuklearmedizin auf nationaler und internationaler Ebene gegenüber Politik und Öffentlichkeit vertritt. Ihre ethischen Werte orientieren sich an der Forderung nach Exzellenz und Integrität wissenschaftlichen Handelns und der daraus abgeleiteten Stellungnahmen sowie am Ziel der Verbesserung der Diagnostik und der Behandlung von Patienten.

Für die Glaubwürdigkeit der DGN gegenüber der Öffentlichkeit sowie der in ihrem Auftrag stets ehrenamtlich agierenden Amts- und Mandatsträger ist ein hohes Maß an ethi- scher Fundierung auf allen Ebenen Voraussetzung. Sie erfordert einen offenen und nachvollziehbaren Umgang mit materiellen und immateriellen Interessen („Sekundärinteressen“), die mit der Forderung nach unvoreingenommener Ausübung eines Mandates in einer wissenschaftlichen Fachgesellschaft in Konflikt treten können.

Mit den Grundsätzen zu Transparenz, Ethik und Vermeidung von Interessenkonflikten und den Verfahrensstandards zur Offenlegung und Bewertung sekundärer Interessen verpflichtet sich die DGN unter anderem

- zu einem transparenten Umgang mit der Industrie,
- zur Erfassung und Bewertung von Sekundärinteressen vor beabsichtigter Übertragung bzw. Übernahme einer Funktion in der DGN,

- zur Offenlegung von Sekundärinteressen der Amts- und Mandatsträger der DGN, ggf. auch der daraus resultierenden Einschränkung hinsichtlich der Ausübung eines Mandats.

Die Grundsätze zu Transparenz, Ethik und Vermeidung von Interessenkonflikten sowie die Verfahrensstandards zur Offenlegung und Bewertung sekundärer Interessen können Sie sich auf der Homepage der DGN unter „Die DGN, Selbstverpflichtung/ Werte“ herunterladen. 